\title{
Severe Neonatal Episodic Laryngospasm
}

National Cancer Institute

\section{Source}

National Cancer Institute. Severe Neonatal Episodic Laryngospasm. NCI Thesaurus.

Code 1122792.

Myotonia caused by mutations of the SCN4A gene, resulting in sodium muscle

channelopathy. It is characterized by severe episodes of laryngospasm in the neonatal period, which can be alleviated by channel blockers. 